\title{
Synthesis of novel nitrogen containing naphtho[2,1-b]furan derivatives and investigation of their anti microbial activities
}

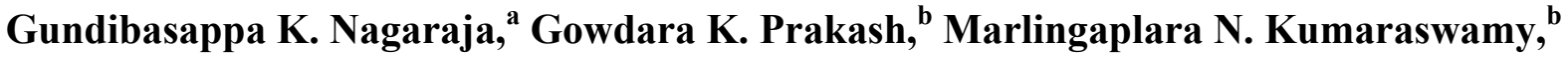 \\ Vijayavittala P. Vaidya, ${ }^{b}$ and Kittappa M. Mahadevan ${ }^{b}$ * \\ ${ }^{a}$ Department of PG Studies and Research in Polymer Science, University of Mysore. Sir M.V. PG \\ Centre, Thubinakere-571 402, Mandya, Karnataka. India \\ ${ }^{b}$ Department of PG Studies and Research in Chemistry, Kuvempu University, Shankaraghatta- \\ 577 451, Shimoga, Karnataka. India \\ E-mail: mahadevanmalavalli@yahoo.co.in
}

\begin{abstract}
Ethyl naphtho[2,1-b]furan-2-carboxylate has been prepared from 2-hydroxy-1- naphthaldehyde 1 and ethyl chloroacetate, which on further treatment with hydrazine hydrate gave naphtha[2,1$b$ ] furan-2-carbohydrazide $\mathbf{3}$. The resulting compound $\mathbf{3}$ was treated with substituted aldehydes of 2-chloro-3-formylquinolines to give the Schiff base, which on treatment with chloro acetyl chloride gave the title compounds. The structure of the compounds has been confirmed by elemental analysis and spectral studies. The synthesized compounds were screened for their antibacterial and antifungal activities.
\end{abstract}

Keywords: Naphtho[2,1-b]furan-2-carbohydrazide, 2-chloro-3-formylquinoline, Schiff base azetidinones, antibacterial, antifungal activity

\section{Introduction}

Naphthofurans possess a broad range of biological activities that are constituents of important natural products. ${ }^{1-3}$ These plant extracts are being used for traditional medicines, ${ }^{4-11}$ and some of them for example mansonone $\mathrm{D},{ }^{12}$ Dunnione, ${ }^{13}$ etc are also vital biologically active agents. Naphthofurans fused or coupled with nitrogen heterocycles do not occur in nature. Even the synthesis of naphthofurans coupled or fused with nitrogen heterocycles are not reported so for, except some reports of such compounds from our laboratory. ${ }^{14-18}$ Since the discovery of penicillin, azetidinone based heterocycles have been an important class of drugs with wide therapeutic activities ${ }^{19-24}$ and the heterocycles containing quionolines moieties also maintain wide range of biological activities. ${ }^{25-28}$ Hence, with these observation we examine the feasibility 
and efficiency of an approach to synthesis naphthofuran coupled with both quinolines and azetidinone $^{29}$ nucleus, which turns to exhibit significant antimicrobial activities.<smiles>O=Cc1c(O)ccc2ccccc12</smiles>

1

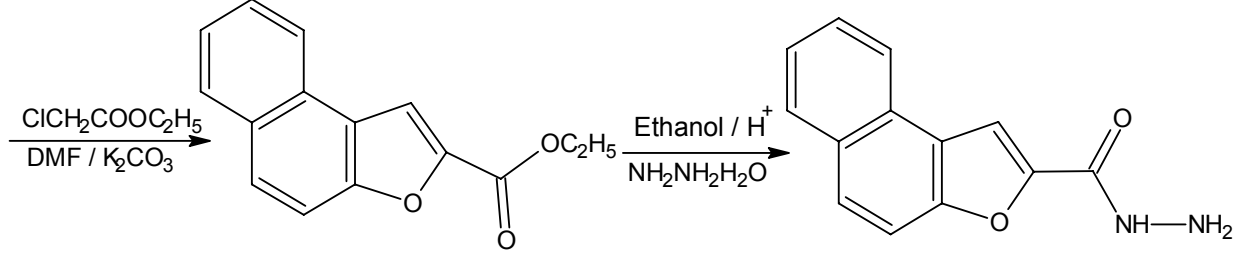

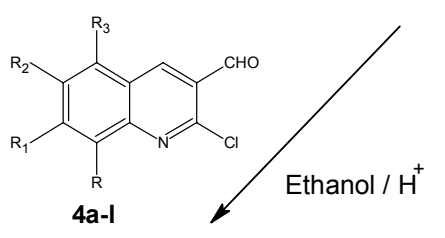<smiles>[R]c1c([R])c([R])c2nc(Cl)c(/C=N/NC(=O)c3cc4c(ccc5ccccc54)o3)cc2c1[R]</smiles><smiles>[R]c1c([R])c([R])c2nc(Cl)c(C3C(Cl)C(=O)N3NC(=O)c3cc4c(ccc5ccccc54)o3)cc2c1[Y]([H])([H])C(=O)Cl</smiles>
$\begin{array}{llll}\mathbf{R} & \mathbf{R}_{1} & \mathbf{R}_{2} & \mathbf{R}_{3}\end{array}$
a. $\begin{array}{lllll}\mathbf{H} & \mathbf{H} & \mathbf{H} & \mathbf{H}\end{array}$
b. $\begin{array}{lllll}\mathbf{H} & \mathrm{H} & \mathrm{CH}_{3} & \mathrm{H}\end{array}$
c. $\mathbf{H}$
$\mathrm{CH}_{3}$
H $\quad \mathbf{H}$
d. $\begin{array}{llll}\mathrm{CH}_{3} & \mathrm{H} & \mathrm{H} & \mathrm{H}\end{array}$
$\begin{array}{lllll}\text { e. } & \mathrm{H} & \mathrm{H} & \mathrm{OCH}_{3} & \mathrm{H}\end{array}$
f. $\begin{array}{llll}\mathrm{H} & \mathrm{OCH}_{3} & \mathrm{H} & \mathrm{H}\end{array}$
g. $\mathrm{OCH}_{3} \mathrm{H} \quad \mathrm{H} \quad \mathrm{H}$
h. $\mathrm{H} \quad \mathrm{H} \quad \mathrm{Br} \quad \mathrm{H}$
$\begin{array}{lllll}\text { i. } & \mathrm{H} & \mathrm{H} & \mathrm{Cl} & \mathrm{H}\end{array}$
$\begin{array}{lllll}\text { j. } & \text { H } & \text { Cl } & \text { H } & \text { H }\end{array}$
$\begin{array}{lllll}\text { k. } & \mathrm{H} & \mathrm{OCH}_{3} & \mathrm{OCH}_{3} & \mathrm{H}\end{array}$
l. $\begin{array}{lllll}\mathrm{H} & \mathrm{OCH}_{3} & \mathrm{OCH}_{3} & \mathrm{OCH}_{3}\end{array}$

Scheme 1. General procedure of $N$-[3-chloro-2-(2-chloroquinolin-3-yl)-4-oxoazetidin-1yl]naphtho[2,1-b]furan-2-carboxamide 6a-1. 


\section{Results and Discussion}

Ethyl naphtho[2,1-b]furan-2-carboxylate 2 was prepared from 2-hydroxy-1-naphthaldehyde on reacting with ethyl chloroacetate in potassium carbonate and DMF in acceptable yields. The identity of the product was determined by IR and ${ }^{1} \mathrm{H}$ NMR spectral studies. The IR spectrum of compound 2 revealed a sharp strong absorption band above $1718 \mathrm{~cm}^{-1}$ due to the presence of the ester function in the structure. The ${ }^{1} \mathrm{H}$ NMR spectra substantiated the results of the IR analysis. The characteristic signals of an ester moiety confirm the presence of ester group in the structure by resonating as quartet and triplet for $\mathrm{CH}_{2}$ and $\mathrm{CH}_{3}$ at $\delta 4.45 \mathrm{ppm}(\mathrm{J}=7 \mathrm{~Hz})$ and $\delta 1.35 \mathrm{ppm}(\mathrm{J}=$ $7 \mathrm{~Hz}$ ) respectively. The aromatic protons resonate as multiplets at $\delta 7.60-8.50 \mathrm{ppm}$, in particular the proton at $\mathrm{C} 5 \& \mathrm{C} 6$ resonate as triplets at $\delta 7.60 \mathrm{ppm}(\mathrm{J}=7 \mathrm{~Hz})$ and $\delta 7.70 \mathrm{ppm}(\mathrm{J}=7 \mathrm{~Hz})$ respectively. $\mathrm{C} 10$ and $\mathrm{C} 11$ resonate as doublet at $\delta 7.90 \mathrm{ppm}(\mathrm{J}=9 \mathrm{~Hz}), \& \delta 8.45 \mathrm{ppm}(\mathrm{J}=8 \mathrm{~Hz})$, $\mathrm{C} 3$ resonate at $\delta 8.50 \mathrm{ppm}$ as singlet and $\mathrm{C} 4 \& \mathrm{C} 7$ at $\delta 8.05-8.15 \mathrm{ppm}$ as a double doublet $(\mathrm{J}=8$ $\mathrm{Hz}$ ) of naphthofuran confirms the structure. Also, its mass spectra revealed a molecular ion peak at $\mathrm{m} / \mathrm{z} 240\left(\mathrm{M}^{+}\right)$corresponding to the molecular formula $\mathrm{C}_{15} \mathrm{H}_{12} \mathrm{O}_{3}$. Thus compound 2 was reacted with hydrazine hydrate under acidic condition in ethanol at reflux temperature to obtain naphtho[2,1-b]furan-2-carbohydrazide $\mathbf{3}$ in good yields. The structure of $\mathbf{3}$ was confirmed by IR, NMR and mass spectral analysis. The IR spectrum of $\mathbf{3}$ showed the absence of ester stretching frequency, instead it gave a band at $1657 \mathrm{~cm}^{-1}$ for carbonyl group and showing two sharp bands in the region of $3300-3400 \mathrm{~cm}^{-1}$ due to $-\mathrm{NH}_{2}$ group and at $3100-3400 \mathrm{~cm}^{-1}$ for $-\mathrm{NH}$ frequencies. ${ }^{1} \mathrm{H}$ NMR spectrum of compound 3 exhibited no peak corresponds to ester instead it shows signals at $\delta 10.12 \mathrm{ppm}$ and $\delta 4.62 \mathrm{ppm}$ for $-\mathrm{NH}$ and $-\mathrm{NH}_{2}\left(\mathrm{D}_{2} \mathrm{O}\right.$ exchangeable $)$ of hydrazide respectively. The structure was further confirmed by recording its mass spectra. It gave the molecular ion peak at $\mathrm{m} / \mathrm{z} 226\left(\mathrm{M}^{+}\right)$corresponds to molecular formula $\mathrm{C}_{13} \mathrm{H}_{10} \mathrm{~N}_{2} \mathrm{O}_{2}$.

To prepare Schiff bases 5a-l, the compound $\mathbf{3}$ was treated with various aromatic aldehydes of 2-chloro-3-formyl quinolines $\mathbf{4 a - 1}$ in presence of catalytic amount of acetic acid in absolute ethanol. The IR spectrum of $\mathbf{5 a}$ exhibit absorption band at $1602 \mathrm{~cm}^{-1}$ due to $-\mathrm{C}=\mathrm{N}$ and amide stretching frequency remain at $1655 \mathrm{~cm}^{-1}$. ${ }^{1} \mathrm{H}$ NMR of 5a exhibits multiplets at $\delta 7.65-8.55 \mathrm{ppm}$ for 12 protons. Whereas imine protons found to resonate at $\delta 8.63 \mathrm{ppm}$ and $-\mathrm{NH}\left(\mathrm{D}_{2} \mathrm{O}\right.$ exchangeable) protons appeared at $\delta 12.21 \mathrm{ppm}$.

Finally, to obtain the title compounds $N$-[3-chloro-2-(2-chloroquinolin-3-yl)-4-oxoazetidin-1yl]naphtho[2,1-b]furan-2-carboxamides 6a-l, the compounds 5a-I were made to react with chloro acetyl chloride and triethylamine in dioxane. The structural analyses of the newly synthesized molecule 6a-I were confirmed by IR, ${ }^{1} \mathrm{H}$ NMR and mass spectral investigations. The IR spectra of the compound 6a revealed two sharp strong absorption bands at $1657 \mathrm{~cm}^{-1}$ and $1709 \mathrm{~cm}^{-1}$ for carbonyl group in azetidinone ring and amide group respectively. Further, ${ }^{1} \mathrm{H}$ NMR spectrum exhibited multiplets in the region at $\delta 7.60-8.90 \mathrm{ppm}$ for 12 aromatic protons. Two protons present in azetidinone ring i.e. $\mathrm{N}-\mathrm{CH}-\mathrm{C}$ and $\mathrm{C}-\mathrm{CH}-\mathrm{Cl}$ are found to resonate as doublets at $\delta 1.8$ ppm and $\delta 2.5 \mathrm{ppm}$ respectively. The structure was further confirmed by its Mass spectral studies. It gave molecular ion peak at $\mathrm{m} / \mathrm{z} 476\left(\mathrm{M}^{+}\right)$corresponds to molecular formula 
$\mathrm{C}_{25} \mathrm{H}_{15} \mathrm{Cl}_{2} \mathrm{~N}_{3} \mathrm{O}_{3}$ (scheme-1). Similarly, all these compounds were purified by column chromatography and characterized on the basis of spectral studies. The spectral details of all the synthesized compounds are given in appropriate place and are in agreement with the assigned structures.

The present protocol describes a simple and efficient method for the synthesis of azetidinones by different Schiff bases of naphtho[2,1-b]furans. It has been demonstrated that cyclocondensation of schiff bases with chloroacetyl chloride in triethyl amine revealed with fairly high yields in a relatively short reaction time and easy work-up procedures. These conditions enable this method to be good protocol for the synthesis of 2-azetidinone based heterocycles.

\section{Experimental Section}

General Procedures. All the reagents were obtained commercially and used with further purification. The melting points were determined on a Kofler hot-stage apparatus and are uncorrected; IR spectra were recorded with a FT Bruker spectrometer in KBr optics. ${ }^{1} \mathrm{H}$ NMR spectra were recorded on a Varian VXR-400 (400 MHz) spectrometer in DMSO-d 6 solutions. The chemical shifts were expressed in the ppm ( $\delta$ scale) downfield from TMS. Microanalysis was determined on a Fisons EA 1108 instrument. Silica gel Merck (60-120 mesh) and DCAlufolien 60 F254 were normally used for column and TLC Chromatography respectively.

\section{Evaluation of antimicrobial activity}

The in vitro antimicrobial activity was carried out against $24 \mathrm{hr}$ old cultures of two bacteria and two fungi by cup-plate method. ${ }^{30}$ Compounds 6a-1 (except 6b, 6d, 6f, 6g, and 6j) has been tested for their antibacterial activity against Pseudomonas aerugenosa and Staphylococcus aureus and antifungal activity against Aspergillus niger and Candida albicans. Nutrient agar and potato dextrose agars were used to culture the bacteria and fungus respectively. The compounds were tested at a concentration of $0.005 \mathrm{~mol} / \mathrm{ml}$ in DMF solution. The solution of Chloramphenicol (2 $\mathrm{mg} / \mathrm{ml})$ and Flucanazole $(2 \mathrm{mg} / \mathrm{ml})$ were prepared in sterilized water and used as standards for comparison of antibacterial and antifungal activities respectively. The compounds were tested at varied concentration. The minimum inhibition concentration was found to be $0.001 \mathrm{~mol} / \mathrm{ml}$ in DMF against all organisms. Inhibition was recorded by measuring the diameter of the inhibition zone at the end of $24 \mathrm{~h}$ for bacteria at $28^{\circ} \mathrm{C}$ and $48 \mathrm{~h}$ for fungus at $35^{\circ} \mathrm{C}$. Each experiment was repeated thrice and the average of the three independent determinations was recorded. The protocols were summarized in (Table 1). The compounds $\mathbf{6 c}$ and $\mathbf{6 e}$ showed promising activity against $P$. aerugenosa and $\mathbf{6 i}, \mathbf{6 l}$ and $\mathbf{6 k}$ against $S$. aureus. The compounds $\mathbf{6 c}$ and $\mathbf{6 l}$ against $A$. niger and $\mathbf{6 c}$ and $\mathbf{6 i}$ against $C$. albicans exhibited significant activity. 
Table 1. Antimicrobial activity of the compounds 6a-I

\begin{tabular}{ccccc}
\hline & \multicolumn{2}{c}{$\begin{array}{c}\text { Antibacterial activity } \\
\text { Zone of inhibition in mm }\end{array}$} & \multicolumn{2}{c}{$\begin{array}{c}\text { Antifungal activity } \\
\text { Zone of inhibition in mm }\end{array}$} \\
\cline { 2 - 5 } Compd. & P.aerugenosa & S. aureus & A. niger & C. albicans \\
\hline 6a & 14 & 14 & 13 & 14 \\
$6 \mathrm{c}$ & 15 & 14 & 20 & 18 \\
$6 \mathrm{e}$ & 18 & 15 & - & 16 \\
$6 \mathrm{~h}$ & - & 15 & 16 & - \\
$6 \mathrm{i}$ & 15 & 18 & 17 & 16 \\
$6 \mathrm{k}$ & - & 16 & 15 & 15 \\
61 & 15 & 16 & 18 & 16 \\
Chloramphenicol & 22 & 24 & - & - \\
Flucanazole & - & - & 25 & 26 \\
\hline
\end{tabular}

Control (DMF) (-) - No activity.

Highly active (inhibition zone $>12 \mathrm{~mm}$ );

Moderately active (inhibition zone 9-12 $\mathrm{mm}$ );

Slightly active (inhibition zone $6-9 \mathrm{~mm}$ );

Inactive - inhibition zone $<6 \mathrm{~mm}$ ).

Ethyl naphtha[2,1-b]furan-2-carboxylate 2. A mixture of 2-Hydroxy naphthaldehyde 1 (1.72 $\mathrm{g}, 10 \mathrm{mmol})$, ethyl chloroacetate $(1.22 \mathrm{~g}, 10 \mathrm{mmol})$ anhydrous potassium carbonate $(25 \mathrm{~g})$ in 25 $\mathrm{ml}$ of dimethyl formamide was heated under reflux for $24 \mathrm{~h}$. After completion of the reaction (TLC), the reaction mixture was poured into ice-cold water. The resultant product separated was filtered off and recrystallized from ethyl acetate gave $\mathbf{2}$ in $80-90 \%$ yields.

Naphtho[2,1-b]furan-2-carbohydrazide 3. The compound $2(2.2 \mathrm{~g}, 10 \mathrm{mmol})$ with hydrazine hydrate $(99 \%),(0.50 \mathrm{~g}, 10 \mathrm{mmol})$ under acidic condition in absolute ethanol $(30 \mathrm{ml})$ were refluxed for 2-3 hrs. After the completion of reaction as evidenced by TLC, the reaction mixture was poured onto crushed ice; the solid mass thus separated out was filtered, washed with water and dried to gave the desired compounds 3 in $80-85 \%$ yields.

$N^{\prime}$-[2-Chloroquinolin-3-yl]methylene]naphtho[2,1-b]furan-2-carbohydrazide 5a-l. Naphtho [2,1-b]furan-2-carbohydrazide $(0.01 \mathrm{~mol}) \mathbf{3}$, 2-chloro-3-formylquinoline $4 \mathbf{a}(0.01 \mathrm{~mol})$ were taken in ethanol with catalytic amount of acetic acid $(20 \mathrm{ml})$ and heated to refluxed for 3-4 hrs. After conclusion of the reaction (TLC), the reaction mixture was poured onto crushed ice; the solid mass thus separated out was filtered, washed with water and dried to give the desired compounds $\mathbf{5 a}$ in $70-75 \%$ yields.

\section{$\mathrm{N}$-[3-Chloro-2-(2-chloroquinolin-3-yl)-4-oxoazetidin-1-yl]naphtho[2,1-b]furan-2-}

carboxamide 6a-l. General Procedure. In a typical example, chloroacetyl chloride $(0.01 \mathrm{~mol})$ in $5 \mathrm{ml}$ of dioxane cooled to $0^{\circ} \mathrm{C}$, to this triethyl amine $(0.01 \mathrm{~mol})$ in $5 \mathrm{ml}$ dioxane was added. To this Schiff's base $5(0.01 \mathrm{~mol})$ in $5 \mathrm{ml}$ dioxane was added and refluxed for $6 \mathrm{hrs}$. After the 
completion of the reaction (monitored by TLC), the reaction mixture poured into ice-cold water to get solid, which was filtered and dried. Thus crude product obtained was purified by column chromatography on silica gel \{eluent: ethyl acetate: petroleum-ether (bp $\left.40{ }^{\circ} \mathrm{C}-60{ }^{\circ} \mathrm{C}\right)=1: 9$ \}.

$\boldsymbol{N}$-[3-Chloro-2-(2-chloroquinolin-3-yl)-4-oxoazetidin-1-yl]naphtho[2,1-b]furan-2-

carboxamide (6a). Brown solid, (67\%), mp 242-243 ${ }^{\circ} \mathrm{C}$, $\mathrm{MS}$ : $\left(\mathrm{M}^{+}\right)$476; Anal.Calcd for $\mathrm{C}_{25} \mathrm{H}_{15} \mathrm{Cl}_{2} \mathrm{~N}_{3} \mathrm{O}_{3}$ : C 63.04, H 3.17, $\mathrm{N}$ 8.82. Found: $\mathrm{C}$ 63.25, H 3.15, N 8.79. IR (KBr, cm $\left.{ }^{-1}\right): 784$ $(\mathrm{C}-\mathrm{Cl}), 1657\left(\mathrm{C}=\mathrm{O}\right.$ azetidinone), $1709(\mathrm{C}=\mathrm{O}), 3420(\mathrm{~N}-\mathrm{H}) ;{ }^{1} \mathrm{H}$ NMR $(\delta, \mathrm{DMSO}): 1.84(\mathrm{~d}, 1 \mathrm{H}, \mathrm{N}-$ CH-C), 2.52 (d, 1H, C-CH-Cl), 7.21-9.25 (m, 12H, Ar-H), 10.45 (s, 1H, CONH), ${ }^{13} \mathrm{C}$ NMR (DMSO) $\delta: 41.12,110.23,112.57,122.43,124.16,126.56,127.23,129.51,130.37,147.42$, $152.43,157.90,166.85$.

N-[2-(6-Methyl-2-chloroquinolin-3-yl)-3-chloro-4-oxoazetidin-1-yl]naphtho[2,1-b]furan-2carboxamide (6b). Brown solid, (72\%), mp 243-245 ${ }^{\circ} \mathrm{C}$, MS: $\left(\mathrm{M}^{+}\right)$490; Anal.Calcd for $\mathrm{C}_{26} \mathrm{H}_{17} \mathrm{Cl}_{2} \mathrm{~N}_{3} \mathrm{O}_{3}$ : C 63.69, H 3.49, N 8.57. Found: C 63.65, H 3.45, N 8.65. IR (KBr, cm $\left.{ }^{-1}\right): 786$ $(\mathrm{C}-\mathrm{Cl}), 1656\left(\mathrm{C}=\mathrm{O}\right.$ azetidinone), $1706(\mathrm{C}=\mathrm{O}), 3422(\mathrm{~N}-\mathrm{H}) ;{ }^{1} \mathrm{H}$ NMR $(\delta, \mathrm{DMSO}): 1.13(\mathrm{~s}, 3 \mathrm{H}$, $\mathrm{CH}_{3}$ ), 1.82 (d, 1H, N-CH-C), 2.54 (d, 1H, C-CH-Cl), 7.23-9.29 (m, 11H, Ar-H), 10.41 (s, 1H, $\mathrm{CONH}),{ }^{13} \mathrm{C}$ NMR (DMSO) $\delta: 36.28,41.11,110.25,112.47,122.46,124.26,126.58,127.13$, $128.59,130.47,147.62,152.33,157.50,167.15$.

N-[2-(5-Methyl-2-chloroquinolin-3-yl)-3-chloro-4-oxoazetidin-1-yl]naphtho[2,1-b]furan-2-

carboxamide (6c). Brown solid, (73\%), mp 241-244 ${ }^{\circ} \mathrm{C}$, MS: $\left(\mathrm{M}^{+}\right)$490; Anal.Calcd for $\mathrm{C}_{26} \mathrm{H}_{17} \mathrm{Cl}_{2} \mathrm{~N}_{3} \mathrm{O}_{3}$ : C 63.69, H 3.49, N 8.57. Found: $\mathrm{C}$ 63.70, H 3.45, N 8.62. IR (KBr, cm $\left.{ }^{-1}\right): 785$ $(\mathrm{C}-\mathrm{Cl}), 1655\left(\mathrm{C}=\mathrm{O}\right.$ azetidinone), $1708(\mathrm{C}=\mathrm{O}), 3423(\mathrm{~N}-\mathrm{H}) ;{ }^{1} \mathrm{H}$ NMR $(\delta, \mathrm{DMSO}): 1.13(\mathrm{~s}, 3 \mathrm{H}$, $\left.\mathrm{CH}_{3}\right), 1.81$ (d, 1H, N-CH-C), 2.53 (d, 1H, C-CH-Cl), 7.23-9.29 (m, 11H, Ar-H), 10.42 (s, 1H, $\mathrm{CONH}),{ }^{13} \mathrm{C}$ NMR (DMSO) $\delta: 36.53,41.32,110.53,112.67,122.13,123.96,126.26,126.63$, $129.55,130.47,146.92,152.41,157.90,166.84$.

N-[2-(4-Methyl-2-chloroquinolin-3-yl)-3-chloro-4-oxoazetidin-1-yl]naphtho[2,1-b]furan-2carboxamide (6d). Brown solid, (72\%), mp 243-245 ${ }^{\circ} \mathrm{C}$, MS: ( ${ }^{+}$) 490; Anal.Calcd for $\mathrm{C}_{26} \mathrm{H}_{17} \mathrm{Cl}_{2} \mathrm{~N}_{3} \mathrm{O}_{3}$ : C 63.69, H 3.49, N 8.57. Found: C 63.65, H 3.45, N 8.65. IR (KBr, cm $\left.{ }^{-1}\right): 786$ $(\mathrm{C}-\mathrm{Cl}), 1653\left(\mathrm{C}=\mathrm{O}\right.$ azetidinone), $1705(\mathrm{C}=\mathrm{O}), 3421(\mathrm{~N}-\mathrm{H}) ;{ }^{1} \mathrm{H}$ NMR $(\delta, \mathrm{DMSO}): 1.14(\mathrm{~s}, 3 \mathrm{H}$, $\mathrm{CH}_{3}$ ), 1.83 (d, 1H, N-CH-C), 2.55 (d, 1H, C-CH-Cl), 7.21-9.35 (m, 11H, Ar-H), 10.43 (s, 1H,

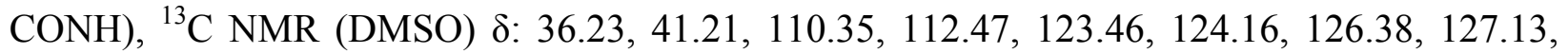
$129.59,130.46,147.72,152.33,157.50,167.35$.

$\boldsymbol{N}$-[2-(6-Methoxy-2-chloroquinolin-3-yl)-3-chloro-4-oxoazetidin-1-yl]naphtho[2,1-b]furan-2carboxamide (6e). Brown solid, (65\%), mp 245-247 ${ }^{\circ} \mathrm{C}, \mathrm{MS}$ : $\left(\mathrm{M}^{+}\right)$506; Anal.Calcd for $\mathrm{C}_{26} \mathrm{H}_{17} \mathrm{Cl}_{2} \mathrm{~N}_{3} \mathrm{O}_{4}$ : C 61.67, H 3.38, N 8.30. Found: C 63.65, H 3.45, N 8.25. IR (KBr, cm $\left.{ }^{-1}\right): 784$ $(\mathrm{C}-\mathrm{Cl}), 1657\left(\mathrm{C}=\mathrm{O}\right.$ azetidinone), $1709(\mathrm{C}=\mathrm{O}), 3420(\mathrm{~N}-\mathrm{H}) ;{ }^{1} \mathrm{H}$ NMR $(\delta, \mathrm{DMSO}): 3.82$ (s, 3H, $\left.\mathrm{OCH}_{3}\right), 1.81$ (d, 1H, N-CH-C), 2.53 (d, 1H, C-CH-Cl), 7.23-9.27 (m, 11H, Ar-H), 10.43 (s, 1H, $\mathrm{CONH}),{ }^{13} \mathrm{C}$ NMR (DMSO) $\delta: 41.22,55.39,110.35,112.67,123.43,124.16,126.26,127.35$, $128.61,130.35,147.45,151.93,157.50,166.75$.

$\mathrm{N}$-[2-(5-Methoxy-2-chloroquinolin-3-yl)-3-chloro-4-oxoazetidin-1-yl]naphtho[2,1-b]furan-2carboxamide (6f). Brown solid, (68\%), mp 246-247 $\mathrm{C}, \mathrm{MS}$ : $\left(\mathrm{M}^{+}\right)$506; Anal.Calcd for 
$\mathrm{C}_{26} \mathrm{H}_{17} \mathrm{Cl}_{2} \mathrm{~N}_{3} \mathrm{O}_{4}$ : C 61.67, H 3.38, N 8.30. Found: C 63.63, H 3.42, N 8.28. IR (KBr, cm $\left.{ }^{-1}\right): 785$ $(\mathrm{C}-\mathrm{Cl}), 1655\left(\mathrm{C}=\mathrm{O}\right.$ azetidinone), $1708(\mathrm{C}=\mathrm{O}), 3423(\mathrm{~N}-\mathrm{H}) ;{ }^{1} \mathrm{H}$ NMR $(\delta, \mathrm{DMSO}): 3.63$ (s, 3H, $\left.\mathrm{OCH}_{3}\right), 1.83$ (d, 1H, N-CH-C), 2.55 (d, 1H, C-CH-Cl), 7.23-9.29 (m, 11H, Ar-H), 10.42 (s, 1H, $\mathrm{CONH}),{ }^{13} \mathrm{C}$ NMR (DMSO) $\delta: 41.32,56.23,110.53,112.67,122.13,123.96,126.26,126.63$, $129.55,130.47,146.92,152.41,157.90,166.84$.

$\mathrm{N}$-[2-(4-Methoxy-2-chloroquinolin-3-yl)-3-chloro-4-oxoazetidin-1-yl]naphtho[2,1-b]furan-2carboxamide (6g). Brown solid, (65\%), mp 245-247 ${ }^{\circ} \mathrm{C}$, MS: ( ${ }^{+}$) 506; Anal.Calcd for $\mathrm{C}_{26} \mathrm{H}_{17} \mathrm{Cl}_{2} \mathrm{~N}_{3} \mathrm{O}_{4}$ : C 61.67, H 3.38, N 8.30. Found: C 63.65, H 3.45, N 8.25. IR (KBr, cm $\left.{ }^{-1}\right): 786$ $(\mathrm{C}-\mathrm{Cl}), 1653\left(\mathrm{C}=\mathrm{O}\right.$ azetidinone), $1705(\mathrm{C}=\mathrm{O}), 3421(\mathrm{~N}-\mathrm{H}) ;{ }^{1} \mathrm{H}$ NMR $(\delta, \mathrm{DMSO}): 3.75$ (s, 3H, $\mathrm{OCH}_{3}$ ), 1.82 (d, 1H, N-CH-C), 2.53 (d, 1H, C-CH-Cl), 7.21-9.35 (m, 11H, Ar-H), 10.43 (s, 1H, CONH), ${ }^{13} \mathrm{C}$ NMR (DMSO) $\delta: 41.21,56.14,110.35,112.47,123.46,124.16,126.38,127.13$, $129.59,130.46,147.72,152.33,157.50,167.35$.

$\mathbf{N}$-[2-(6-Bromo-2-chloroquinolin-3-yl)-3-chloro-4-oxoazetidin-1-yl]naphtho[2,1-b]furan-2carboxamide (6h). Brown solid, (68\%), mp 255-257 ${ }^{\circ} \mathrm{C}, \mathrm{MS}$ : $\left(\mathrm{M}^{+}\right)$555; Anal.Calcd for $\mathrm{C}_{25} \mathrm{H}_{14} \mathrm{BrCl}_{2} \mathrm{~N}_{3} \mathrm{O}_{3}$ : C 54.08, H 2.54, N 7.57. Found: C 53.98, H 2.45, N 7.65. IR ( $\left.\mathrm{KBr}, \mathrm{cm}^{-1}\right): 784$ $(\mathrm{C}-\mathrm{Cl}), 1657\left(\mathrm{C}=\mathrm{O}\right.$ azetidinone), $1709(\mathrm{C}=\mathrm{O}), 3420(\mathrm{~N}-\mathrm{H})$; ${ }^{1} \mathrm{H}$ NMR $(\delta, \mathrm{DMSO}): 1.83(\mathrm{~d}, 1 \mathrm{H}, \mathrm{N}-$ $\mathrm{CH}-\mathrm{C}), 2.55$ (d, 1H, C-CH-Cl), 7.21-9.25 (m, 11H, Ar-H), 10.45 (s, 1H, CONH), ${ }^{13} \mathrm{C}$ NMR (DMSO) $\delta: 41.12,110.23,112.57,122.43,124.16,126.56,127.23,129.51,130.37,147.42$, $152.43,157.90,166.85$.

N-[2-(6-Chloro-2-chloroquinolin-3-yl)-3-chloro-4-oxoazetidin-1-yl]naphtho[2,1-b]furan-2carboxamide (6i). Brown solid, (72\%), mp 253-255 $\mathrm{C}, \mathrm{MS}$ : $\left(\mathrm{M}^{+}\right)$510; Anal.Calcd for $\mathrm{C}_{25} \mathrm{H}_{14} \mathrm{Cl}_{3} \mathrm{~N}_{3} \mathrm{O}_{3}$ : C 58.79, $\mathrm{H} 2.76, \mathrm{~N}$ 8.23. Found: C 58.98, H 2.75, N 8.15. IR (KBr, cm $\left.{ }^{-1}\right): 785$ $(\mathrm{C}-\mathrm{Cl}), 1655\left(\mathrm{C}=\mathrm{O}\right.$ azetidinone), $1708(\mathrm{C}=\mathrm{O}), 3423(\mathrm{~N}-\mathrm{H}) ;{ }^{1} \mathrm{H}$ NMR $(\delta, \mathrm{DMSO}): 1.81(\mathrm{~d}, 1 \mathrm{H}, \mathrm{N}-$ CH-C), 2.54 (d, 1H, C-CH-Cl), 7.23-9.29 (m, 11H, Ar-H), 10.42 (s, 1H, CONH), ${ }^{13} \mathrm{C}$ NMR (DMSO) $\delta: 41.32,110.53,112.67,122.13,123.96,126.26,126.63,129.55,130.47,146.92$, $152.41,157.90,166.84$.

N-[2-(5-Chloro-2-chloroquinolin-3-yl)-3-chloro-4-oxoazetidin-1-yl]naphtho[2,1-b]furan-2-

Carboxamide (6j). Brown solid, (75\%), mp 252-252 ${ }^{\circ} \mathrm{C}$, MS: $\left(\mathrm{M}^{+}\right)$510; Anal.Calcd for $\mathrm{C}_{25} \mathrm{H}_{14} \mathrm{Cl}_{3} \mathrm{~N}_{3} \mathrm{O}_{3}$ : C 58.79, H 2.76, N 8.23. Found: C 58.95, H 2.79, N 8.25. IR (KBr, cm $\left.{ }^{-1}\right): 786$ $(\mathrm{C}-\mathrm{Cl}), 1653\left(\mathrm{C}=\mathrm{O}\right.$ azetidinone), $1705(\mathrm{C}=\mathrm{O}), 3421(\mathrm{~N}-\mathrm{H}) ;{ }^{1} \mathrm{H}$ NMR $(\delta, \mathrm{DMSO}): 1.84(\mathrm{~d}, 1 \mathrm{H}, \mathrm{N}-$ $\mathrm{CH}-\mathrm{C}), 2.53$ (d, 1H, C-CH-Cl), 7.21-9.35 (m, 11H, Ar-H), 10.43 (s, 1H, CONH), ${ }^{13} \mathrm{C}$ NMR (DMSO) $\delta: 41.21,110.35,112.47,123.46,124.16,126.38,127.13,129.59,130.46,147.72$, $152.33,157.50,167.35$.

$\mathrm{N}$-[2-(5,6-Dimethoxy-2-chloroquinolin-3-yl)-3-chloro-4-oxoazetidin-1-yl]naphtho[2,1-

b]furan-2-carboxamide (6k). Bown solid, (72\%), mp 265-267 ${ }^{\circ} \mathrm{C}$, MS: ( $\mathrm{M}^{+}$) 536; Anal.Calcd for $\mathrm{C}_{25} \mathrm{H}_{19} \mathrm{Cl}_{2} \mathrm{~N}_{3} \mathrm{O}_{5}$ : C 60.46, H 3.57, N 7.83. Found: C 60.42, H 3.55, N 7.85. IR (KBr, cm $\left.{ }^{-1}\right): 785$ $(\mathrm{C}-\mathrm{Cl}), 1655\left(\mathrm{C}=\mathrm{O}\right.$ azetidinone), $1708(\mathrm{C}=\mathrm{O}), 3423(\mathrm{~N}-\mathrm{H}) ;{ }^{1} \mathrm{H}$ NMR $(\delta, \mathrm{DMSO}): 1.82(\mathrm{~d}, 1 \mathrm{H}, \mathrm{N}-$ CH-C), 2.52 (d, 1H, C-CH-Cl), 3.75 (s, 3H, $\left.\mathrm{OCH}_{3}\right), 3.83$ (s, 3H, OCH $), 7.21-9.35$ (m, 10H, Ar$\mathrm{H}), 10.43$ (s, 1H, CONH), ${ }^{13} \mathrm{C}$ NMR (DMSO) $\delta: 41.21,56.53,110.35,112.47,123.46,124.16$, $126.38,127.13,129.59,130.46,147.72,152.33,157.50,167.35$, 


\section{$\mathrm{N}$-[2-(5,6,7-Trimethoxy-2-chloroquinolin-3-yl)-3-chloro-4-oxoazetidin-1-yl]naphtho[2,1-}

b]furan-2-carboxamide (61). Brown solid, (73\%), mp 268-269 ${ }^{\circ} \mathrm{C}$, MS: $\left(\mathrm{M}^{+}\right)$566; Anal.Calcd for $\mathrm{C}_{28} \mathrm{H}_{21} \mathrm{Cl}_{2} \mathrm{~N}_{3} \mathrm{O}_{6}$ : C 59.38, $\mathrm{H}$ 3.74, $\mathrm{N}$ 7.42. Found: $\left.\mathrm{C} 60.01, \mathrm{H} \mathrm{3.76,} \mathrm{N} \mathrm{7.45.} \mathrm{IR} \mathrm{(KBr,} \mathrm{cm}^{-1}\right)$ : $784(\mathrm{C}-\mathrm{Cl}), 1657\left(\mathrm{C}=\mathrm{O}\right.$ azetidinone), $1709(\mathrm{C}=\mathrm{O}), 3420(\mathrm{~N}-\mathrm{H}) ;{ }^{1} \mathrm{H}$ NMR $(\delta$, DMSO$): 1.82(\mathrm{~d}$, $1 \mathrm{H}, \mathrm{N}-\mathrm{CH}-\mathrm{C}), 2.53$ (d, 1H, C-CH-Cl), $3.63\left(\mathrm{~s}, 3 \mathrm{H}, \mathrm{OCH}_{3}\right), 3.70\left(\mathrm{~s}, 3 \mathrm{H}, \mathrm{OCH}_{3}\right), 3.83(\mathrm{~s}, 3 \mathrm{H}$, $\mathrm{OCH}_{3}$ ), 7.23-9.29 (m, 9H, Ar-H), 10.42 (s, 1H, CONH), ${ }^{13} \mathrm{C}$ NMR (DMSO) $\delta: ~ 41.32,56.31$, $110.53,112.67,122.13,123.96,126.26,126.63,129.55,130.47,146.92,152.41,157.90,166.84$,

\section{Acknowledgements}

The authors are indebted to Professor and Chairman, Department of PG Studies and Research in Chemistry, Kuvempu University for providing laboratory facilities. The authors are greatly thankful to the head, RSIC, Indian Institute of Science, Bangalore for spectral data.

\section{References}

1. Price, J. R.; Robinson, R. J. Chem. Soc. 1940, 1493.

2. Stochigt, J.; Srocka, U.; Zenk, M. H. Phytochemistry 1973, 12, 2389.

3. Inoue, K.; Ueda, S.; Nayeshiro, H.; Inouye, H. Phytochemistry 1982, 22, 737.

4. Ruedi, P.; Eugaster, C. H. Helv. Chem. Acta. 1977, 60, 945.

5. Fujimoto, Y.; Eguchi, T.; Murasaki, C.; Ohashi, Y.; Kakinuma, K.; Takagaki, H.; Abe, M.; Inazawa, K.; Yamazaki, K.; Ikekawa, N.; Yoshikawa, O.; Ikekawa, T. J. Chem. Soc.,Perkin Trans.11991, 2323.

6. Rohatgi, B. K.; Gupta, R. B.; Roy, D.; Khanna, R. N. Indian. J. Chem. 1983, 22B, 886.

7. Khanna, R. N.; Sharma, P. K.; Thomson, R. H. J. Chem. Soc. Perkin Trans.11987, 1882.

8. Sharma, P. K.; Gupta, P. K.; Khanna, R. B.; Rohatgi, B. K. Indian. J. Chem. 1985, 24B, 1070 .

9. Sharma, P. K.; Khanna, R. N.; Rohatgi, B. K.; Thomson, R. H. Phytochemistry1988, 27(2), 633.

10. Ihoue, K.; Ihouye, H.; Chem, C.C.; Phytochemistry 1971, 10, 1603.

11. Briggs, L. H.; Briggs, L. R.; King, A. W. N. Z. J. Sci. 1975, 18, 559.

12. Bettolo, G. M. B.; Cassinovi, C. G.; Galleffi, C. Tetrahedron. Lett. 1965, 4857.

13. Price, J. R.; Robinson, R. J. Chem. Soc. 1939, 1522.

14. Mahadevan, K. M.; Basavaraj Padmashali, Vaidya, V. P. Indian. J. Heterocyclic. Chem. 2001, 11,15 .

15. Nagaraja, G. K.; Kumaraswamy, M. N.; Vaidya, V. P.; Mahadevan, K. M. Arkivoc. 2006, (x) 211 .

16. Mahadevan, K. M.; Vaidya, V. P.; Vagdevi, H. M. Indian. J. Chem. 2003, 42B, 1931. 
17. Mahadevan, K. M.; Basavaraj, K. M.; Prathima Mathias, D. A.; Vaidya, V. P. Indian. J. Chem. 2005, 44B, 773.

18. Mahadevan, K. M.; Vaidya, V. P. Indian. J. Pharm. Sci. 2003, 65, 128.

19. Koniya, T.; Lres Lab, Suiisawa Pharma Co. Ltd, Osaka (Japan) Kugaku No Ryoiki Zokon. 1946, 112, 29. Chem Abstr. 1977, 86, 1656a.

20. Maffii, G.; (Lepetit S P A, Milan Italy), Farmaco (Pavia) Ed Sci. 14, 1954, 176; Chem Abstr. 53, 1959, 20553B.

21. Shrenik Shah, K.; Barker L Peter, Eur Pat Appl. Ep 360. 1986; Chem Abstr. 1989, 110, 173037a.

22. Cooper, R.D. G., Eur. Pat. Appl. Ep. 1988, 252, 744;Chem Abstr. 1989, 110, 74934.

23. Firestone, R. A.; Barker, P. L.; Pisano, J. M.; Ashe, B. M.; Dahlgren, M. E.; Tetrahedran 1990, 46, 2255.

24. Bose, A. K.; Mgnns, M. S.; Kapur, J. C.; Sharma, S. D. J. Indian. Chem. Soc. 1974, 541, 1715.

25. Johnson, J. V.; Rauckman, S.; Baccanari, P. D.; Roth, B. J. Med. Chem. 1989, 32, 1942.

26. Carling, R. W.; Leeson, P. D.; Moseley, A. M.; Baker, R.; Foster, A. C. J. Med. Chem. 1992, $35,1942$.

27. Kokwaro, G. O.; Taylor, G. Drug Chem Toxicol. 1990, 13, 347.

28. Kimura, T.; Imanishi, S.; Arita, M. J. Cardiovasc Pharmacol. 1998, 13, 767.

29. Patel, H. S.; Desai, H. D.; E-Journal of Chemistry, 2004, 1, 194.

30. Sandane, A. R.; Rudresh, K.; Satyanarayan, N. D.; Hiremath, S. P. Indian. J. Pharm. Sci. 1998, 60, 379 . 